\title{
QUALIFICATION OF ANC9 GENERIC INSERT METHODOLOGY (GIM)
}

\author{
Baocheng Zhang*, Boyan D. Ivanov, Kevin Hoskins, Harish Huria, and \\ Andre Luiz Pereira Rebello Jr \\ Westinghouse Electric Company \\ 1000 Westinghouse Dr, Cranberry Township, Pennsylvania, USA \\ zhangb@westinghouse.com, ivanovbd@westinghouse.com, hoskinkc@westinghouse.com, \\ huriahc@westinghouse.com, pereira@westinghouse.com
}

\begin{abstract}
Discrete burnable poisons like the Wet Annular Burnable Absorber and Pyrex have been used in PWR core to improve core power distribution and provide more negative moderator temperature coefficient. The burnable absorber in the burnable poison rods burns fast and is completely gone after one cycle exposure in the core. Traditionally, the burnable poison rods are designed to be part of the fuel assembly. They are burned together with the fuel in the first loading cycle and discharged after one cycle. In recent years, different insertion scenarios of the burnable poison rods have been introduced in the PWR plants operation to improve the fuel performance, for instance, fresh burnable poison rods are inserted into a burned assembly; burned burnable poison rods stay in the original assembly or are replaced in a different assembly.

A Generic Insert Methodology [1] was developed in Westinghouse and implemented in NEXUS/ANC9 code system. With this new methodology, ANC9 is able to follow the assembly history and model all types of absorber insert components for all kinds of insertion scenarios.

An extensive methods validation and qualification effort has been completed by modeling different insertion cases. This paper provides details of the qualification cases along with the analysis of the results.
\end{abstract}

KEYWORDS: GIM, NEXUS, ANC9, depletion, relative strength

\section{INTRODUCTION}

Different absorber components have been designed and used in Pressurized Water Reactors (PWRs) to compensate for core excess reactivity, to maintain the desired state of core reactivity and to reduce neutron leakage. Among these absorber components, some of them are integrated into the fuel, such as IFBA rods and Gd rods. Others are separable from the fuel, e.g. control rods and discrete burnable absorbers like the Wet Annular Burnable Absorber (WABA) and PYREX. All the separable absorber components are called "inserts" in this paper.

${ }^{*}$ Corresponding author

C2019 Westinghouse Electric Company LLC All Rights Reserved 
Based on the function and operation strategy of different absorber components, these inserts can be categorized into two types. The first type is completely moveable, like the control rods, which can be inserted or withdrawn in/from the core whenever needed during a cycle. The second type is restricted during reactor operation and the insertion status can be changed only during fuel shuffling. The typical example of the second type is discrete burnable absorber, i.e. burnable poison (BP). Based on the type of the absorber components and the usage, Westinghouse PWR core design code system NEXUS/ANC9 [2][3] adopts two different methodologies to model the different inserts, i.e. control rod (CR) method and BP method.

Traditionally, the BP rods are designed to be part of the fuel assembly to reduce core critical boron level at the beginning of cycle as well as compress the local power distribution. This means that the fuel and BP rods are burned together from fresh in the first loading cycle. After one cycle exposure in the core, the absorber isotope(s) in the BP rods are completely depleted. Therefore, in most reload designs, after the first cycle, the BP rods will be removed, and the fuel assembly will be loaded again in the core for the next cycle without the BP rods. Occasionally, plant may leave the burned BP rods in the fuel as the water displacement. The BP method in NEXUS/ANC9 was developed to handle this traditional type of BP insertion scenario.

In addition, there are CRs that can be inserted into a fuel assembly at any time at any fuel burnup condition whenever the operation needs. CR insertion during normal operation is one operational feature in the AP1000 ${ }^{1}{ }^{1}$ plant of Westinghouse next generation PWR design. CR depletion functionality was introduced in NEXUS/ANC9 code system. The CR method in NEXUS/ANC9 can track the burnable absorbers in the CRs and capture the impact of CR insertion on the assembly averaged cross-sections for both fresh and burned CRs.

In contrast to the traditional discrete BA utilization, there are cases where the burned BP rods may be occasionally re-inserted into a fresh or burned fuel assembly, or fresh BP could be inserted into a burned fuel assembly to enhance the fuel assembly performance. This special design brings challenges to any code system with once through cross-section methodology since the required assembly cross-section data cannot be generated upfront without knowing the insert types and fuel history when the insertion occurs.

In view of these special discrete BA insertion scenarios, a Generic Inserts Method (GIM) has been developed in Westinghouse Electric Company and implemented in NEXUS/ANC9 code system.

\section{BRIEF DESCRIPTION OF GENERIC INSERT METHODOLOGY (GIM)}

Reviewing the special BP insertion scenarios and comparing them against $\mathrm{CR}$ usage brings out the fact that such a BP insertion is basically a simplified CR case where insertion and withdrawal happen only at the beginning and end of the operation cycle while the local burnup could be arbitrary based on the local power history.

However, there is a significant difference between the use of BP and CRs. As mentioned above, the absorber material of the BP will completely burn out after one cycle in the core. However, the CRs usually deplete extremely slowly. Also, for safety and controllability reason, plant won't allow the control rods heavily burned before discharging them. So CR methodology focused on a area that the absorber inserts have nonsignificant change of the absorption strength.

\footnotetext{
${ }^{1} \mathrm{AP1000}$ is a registered trademark of Westinghouse Electric Company LLC in the United States and may be registered in other countries throughout the world. All rights reserved. Unauthorized use is strictly prohibited.
} 
In view of this, the Generic Insert Methodology is developed by improving the existing ANC9 CR methodology.

In the standard ANC9 CR methodology, the instantaneous CR correction for a rodded fuel node is applied to the assembly average macroscopic cross-sections as well as to the microscopic cross-sections of the tracked isotopes (actinides and fission products). For a depleted CR bank and based on the CR relative strength (or fraction remaining), a CR depletion correction is introduced as the second order correction to capture the CR depletion impact. This CR depletion correction is only applied to the macroscopic crosssections because the $\mathrm{CR}$ relative strength does not change much during its life.

In contrast, the fresh burned BP brings a significantly different local neutron spectrum from that of highly burned BP, and this difference leads to a non-negligible impact on both assembly average macroscopic and isotopic microscopic cross-sections. Thus, like CR depletion correction, the insert depletion effect is expressed as a function of the insert relative strength. GIM model extends the insert (including CR) depletion correction to both macroscopic and microscopic cross-sections, as well as other assembly parameters and pin-wise data.

The absorber insert has both direct and indirect impacts on the fuel assembly cross-sections. The direct impact is from the absorption of insert absorber isotopes, while indirect impact represents the local spectrum and heterogeneity change due to the insertion of absorber material. The direct impact of a generic insert is the direct contribution of the insert absorber isotopes, i.e.:

$$
\Delta \Sigma_{x, g}^{G I, \text { direct }}=\sum_{i \in G I} N^{i} \sigma_{x, g}^{i}
$$

The indirect insert correction is pre-generated through branch calculations and tabulated as a function of insert relative strength. Through tracking the number densities of the insert absorber isotopes, ANC9 can compute the insert relative strength by comparing them against the initial values as:

$$
s=\frac{\sum_{i \in G I} N^{i} w^{i}}{\sum_{i \in G I} N_{0}^{i} w^{i}}
$$

Where the weighting factor $w^{i}$ represents the importance of the absorber isotope " $i$ ". With isotope number densities and the insert relative strength, ANC9 can easily obtain both direct and indirect insert corrections to the nodal cross-sections.

As an enhancement of CR methodology, GIM is replacing the current ANC9 CR model and will apply to all removable absorber insert types including CR.

Like CR insertion, the insertion of BP rods brings local spectrum and heterogeneity changes, which impact the local pin power distribution and the impact accumulates with fuel depletion. This was a significant challenge to the conventional pin power methodology [4]. This issue has been resolved in ANC9 with new pin power methodology [5] by following individual fuel rod history explicitly along with the fuel rod history correction to both pin cell cross-sections and flux form factors.

\section{QUALIFICATION OF GENERIC INSERT METHODOLOGY}

Generic Insert Methodology implemented in NEXUS/ANC9 is used to model both CRs and BP rods. To validate the new methodology and quantify the accuracy in modeling different BP insertion scenarios, several test cases were setup, which cover different fuel assembly types and multiple insert configurations. 
A few sample results are reported in this paper. The qualification focuses on the prediction of both fuel reactivity and pin power distributions.

\subsection{Case Description}

As a fundamental methods validation, single assembly models were generated with different absorber insert types and insertion scenarios. When inserting fresh BP rods into a burned fuel assembly, the assembly initially may or may not have BP rods. Therefore, the two fuel assemblies, respectively with and without WABA rods initially, were modeled to validate the GIM methodology.

Insertion case 1:

- Deplete non-BP assembly, insert 8 WABA rods at 10, 20, and 30 GWD/MTU respectively, and continue depleting the assembly till $60 \mathrm{GWD} / \mathrm{MTU}$

- Repeat the above calculations with 12 and 20 WABA rods instead

Insertion case 2:

- Deplete BP assembly with 20 WABA rods, and replace the burned WABA with 8 fresh WABA rods at 10,20 , and $30 \mathrm{GWD} / \mathrm{MTU}$, respectively, and continue depleting the assembly till $60 \mathrm{GWD} / \mathrm{MTU}$

All single assembly cases were calculated using both ANC9 and PARAGON [6], and PARAGON results were taken as the references.

\subsection{Evaluation of results}

Figures 1 to 5 demonstrate the $k_{\infty}$ difference and pin power Root Mean Square (RMS) and peak power differences between ANC9 and PARAGON for the assembly initially having no BP, while the results comparisons for BP replacement are displayed in Figures 6 to 8 .

As shown in the figures, ANC9 predicted $k_{\infty}$ is in an excellent agreement with reference results throughout the fuel life. The maximum reactivity error is within $100 \mathrm{pcm}$ for all tested cases.

Moreover, ANC9 adopted Pseudo Pin-by-Pin Calculation (P3C) methodology in pin power calculations, it can follow the true history of each individual fuel pin and capture the history effect of burnable absorber insertion. As shown in the figures, ANC9 precisely reproduces PARAGON pin power results. The error of the peaking factors is within $1 \%$.

As noticed from Figure 6, after BP rods replacement and at burnup around $13000 \mathrm{MWD} / \mathrm{MTU}$, the dramatical change of pin power error with depletion is unexpected. This is an off-normal behavior between 12000 and $15000 \mathrm{MWD} / \mathrm{MTU}$ although the pin power error is still within the acceptance criteria. Further investigations found that the error comes from numerical noise when pre-generating the insert depletion correction coefficients. This numeric issue can be easily resolved by changing the definition of the insert depletion correction factor. 

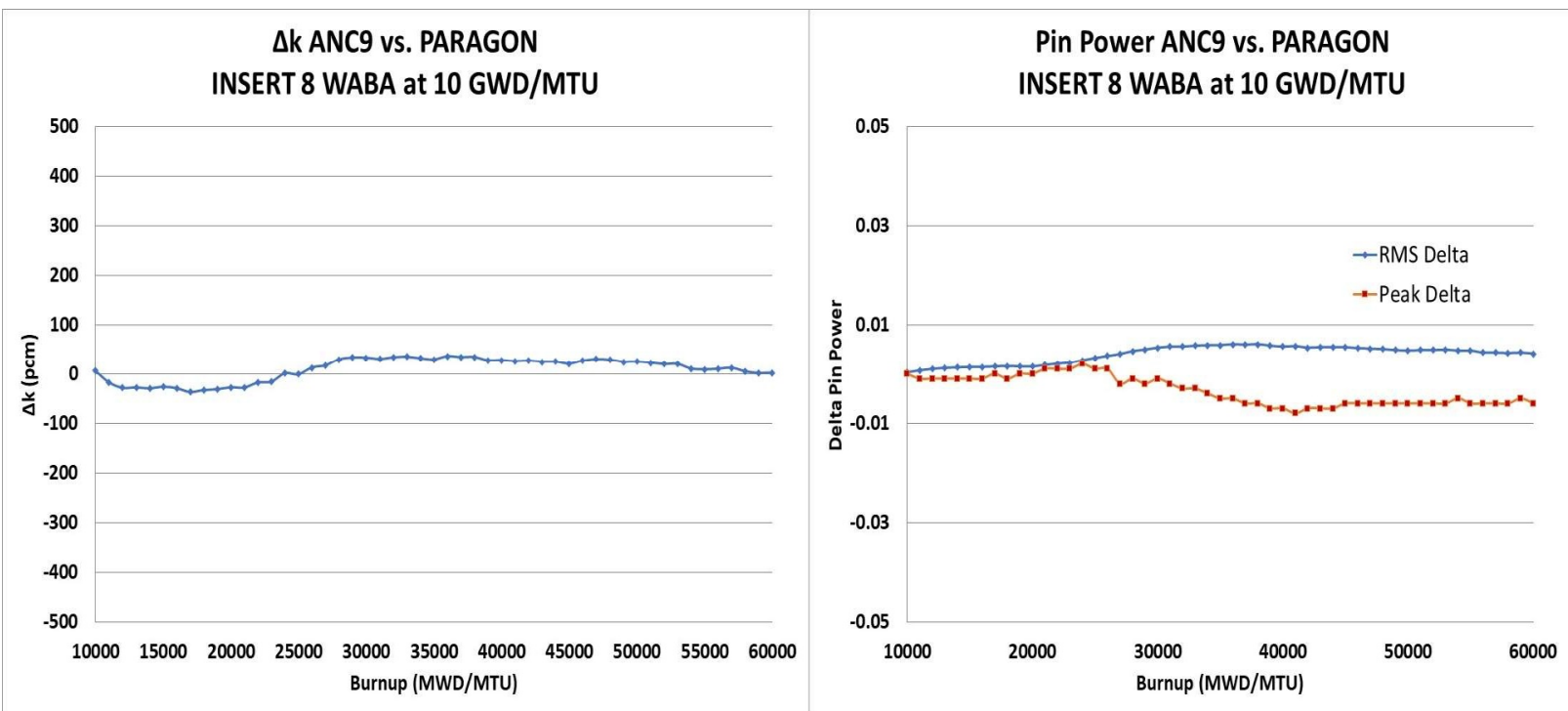

Figure 1. Comparison of eigenvalue and pin power for $8 \mathrm{WABA}$ rods inserted at $10 \mathrm{GWD} / \mathrm{MTU}$
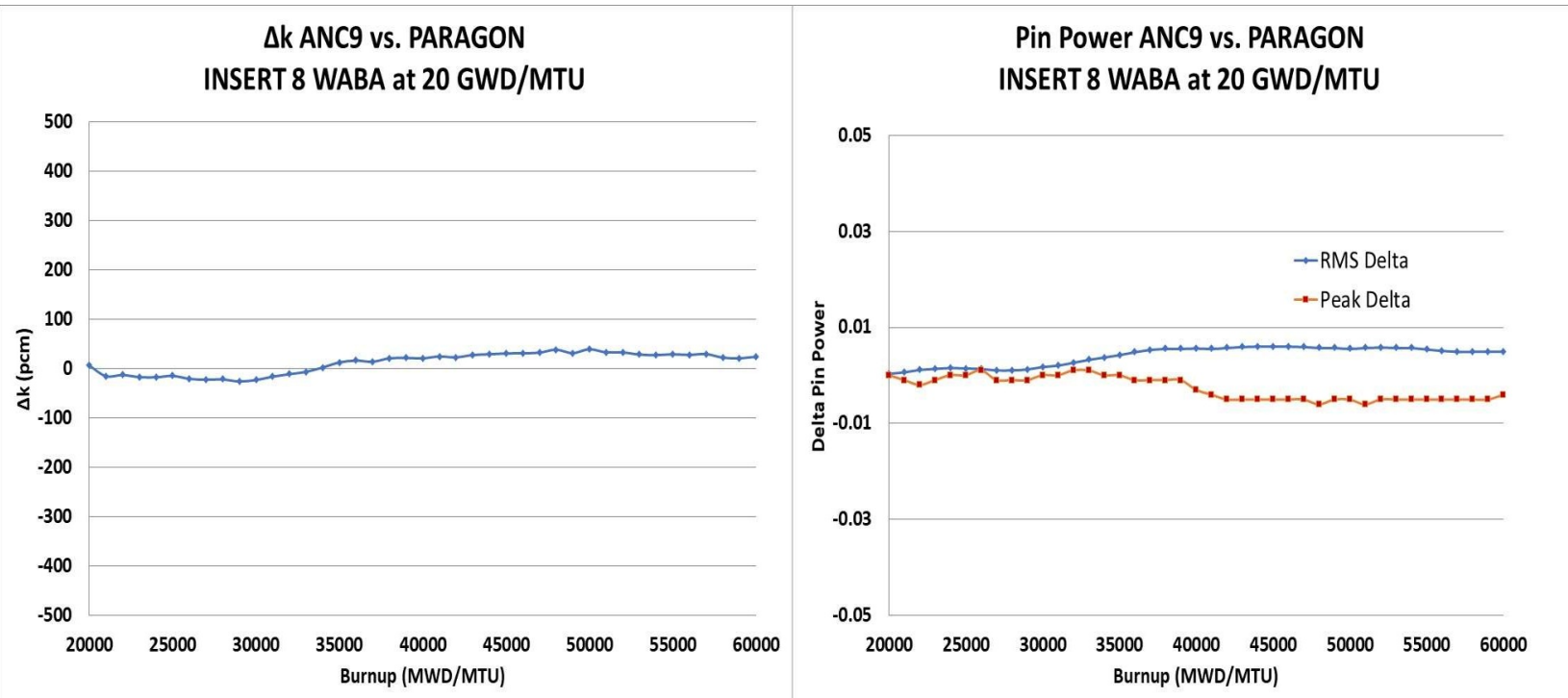

Figure 2. Comparison of eigenvalue and pin power for 8 WABA rods inserted at $20 \mathrm{GWD} / \mathrm{MTU}$ 

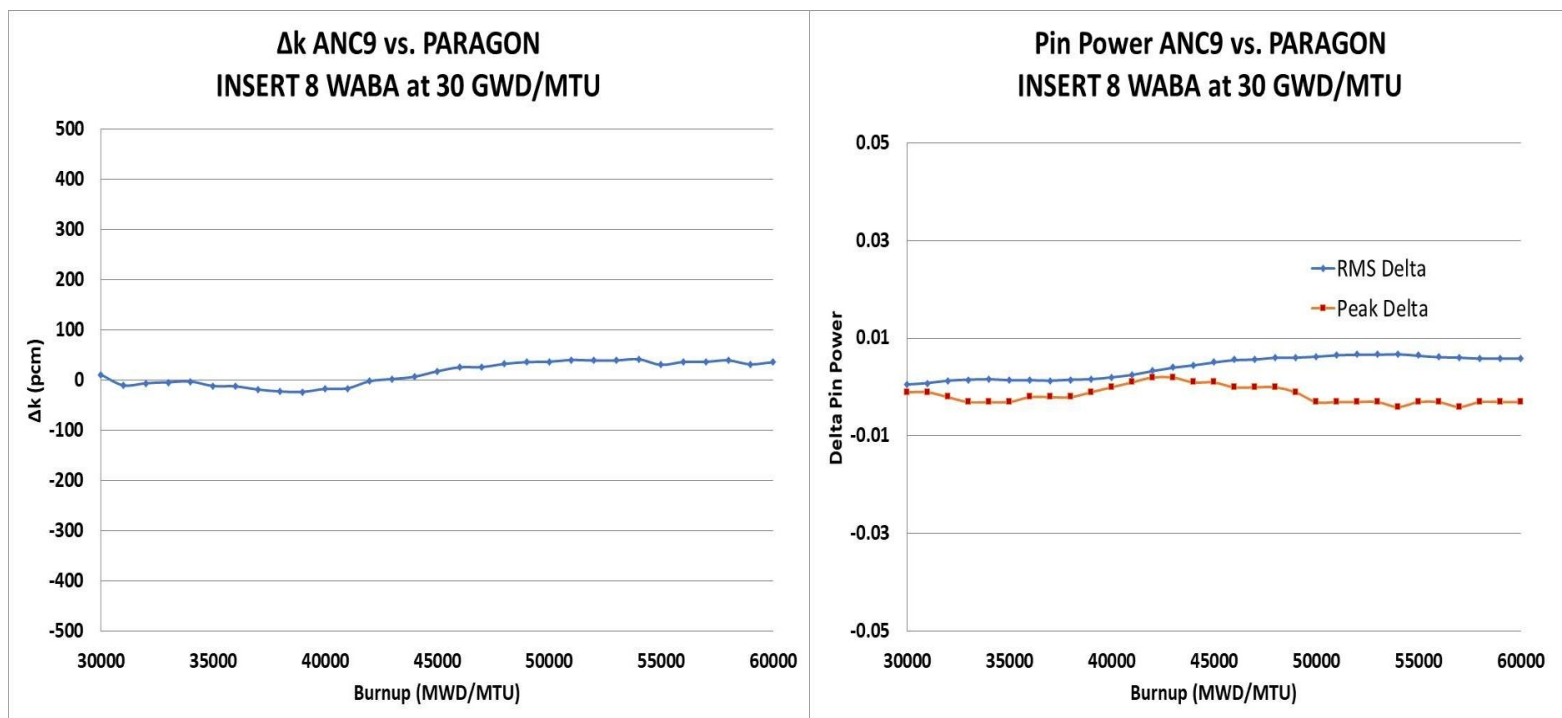

Figure 3. Comparison of eigenvalue and pin power for 8 WABA insert at $30 \mathrm{GWD} / \mathrm{MTU}$
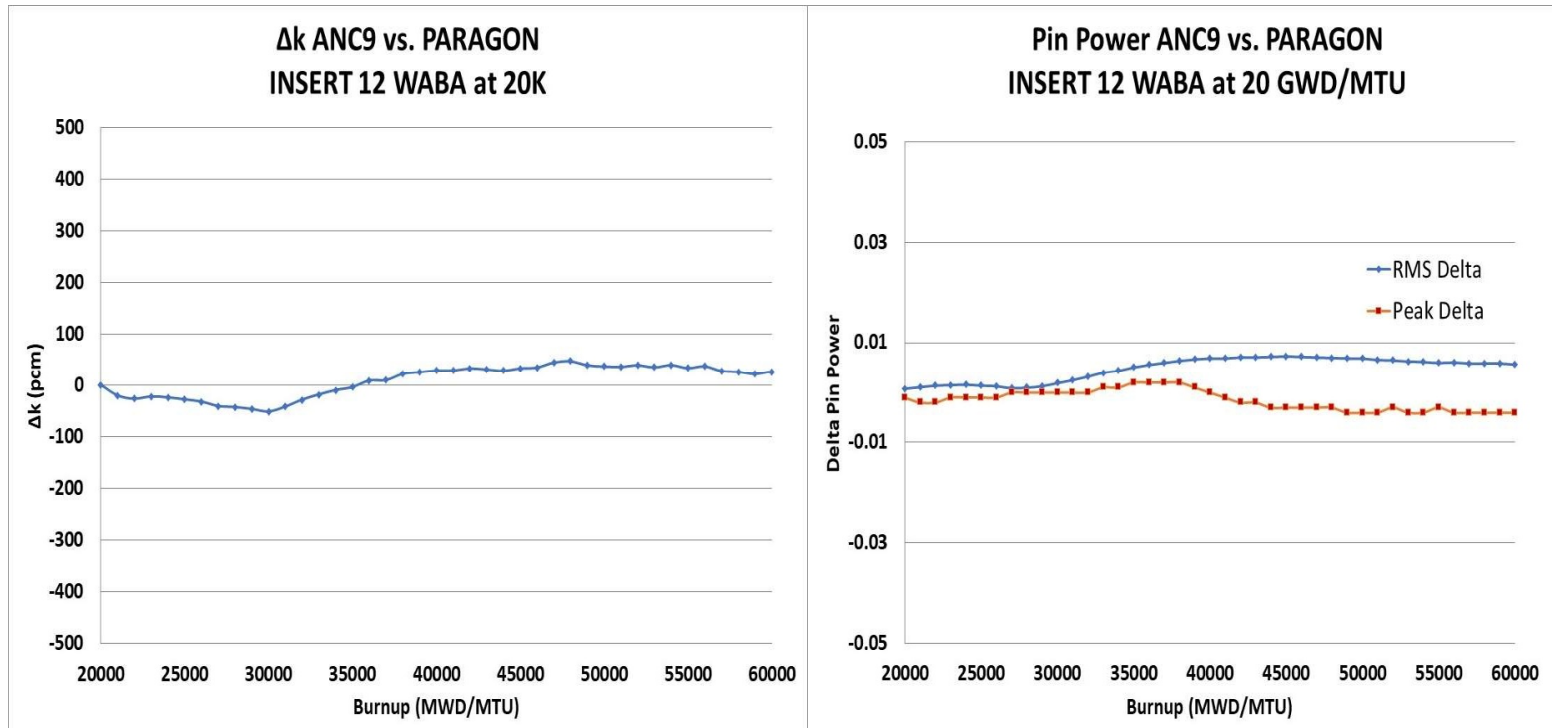

Figure 4. Comparison of eigenvalue and pin power for $12 \mathrm{WABA}$ rods inserted at $20 \mathrm{GWD} / \mathrm{MTU}$ 

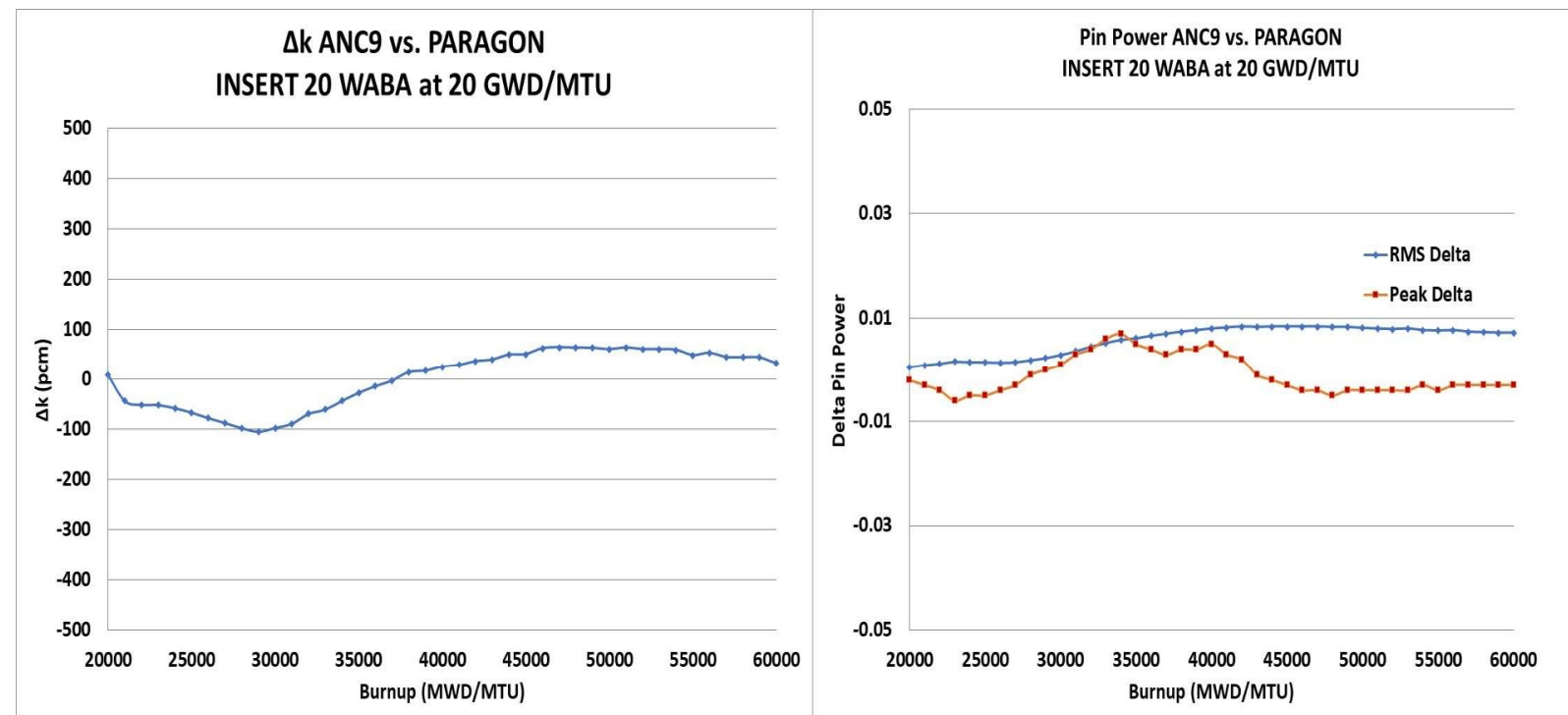

Figure 5. Comparison of eigenvalue and pin power for $20 \mathrm{WABA}$ rods inserted at $20 \mathrm{GWD} / \mathrm{MTU}$
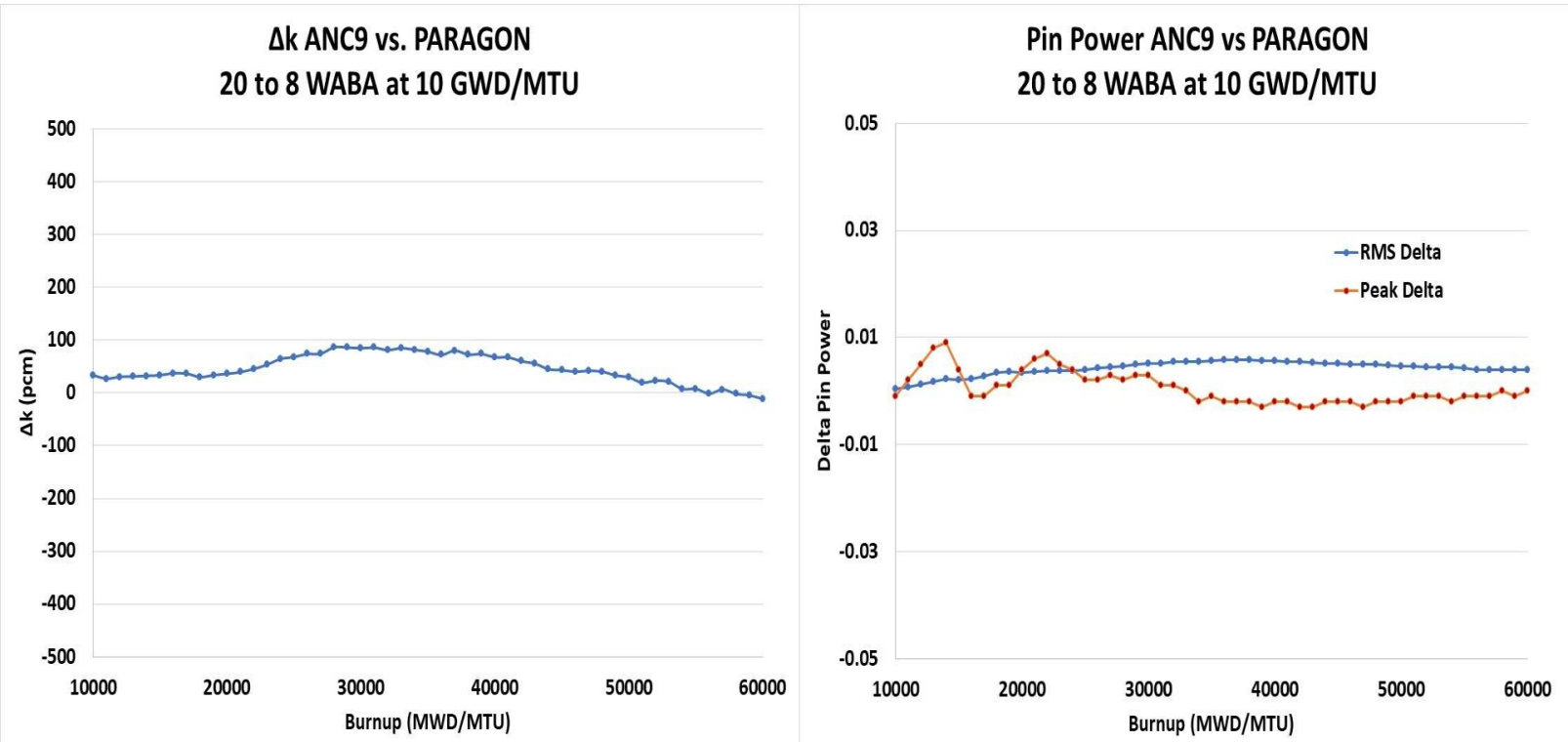

Figure 6. Comparison of eigenvalue and pin power for WABA replacement from 20 to 8 rods at 10 GWD/MTU 

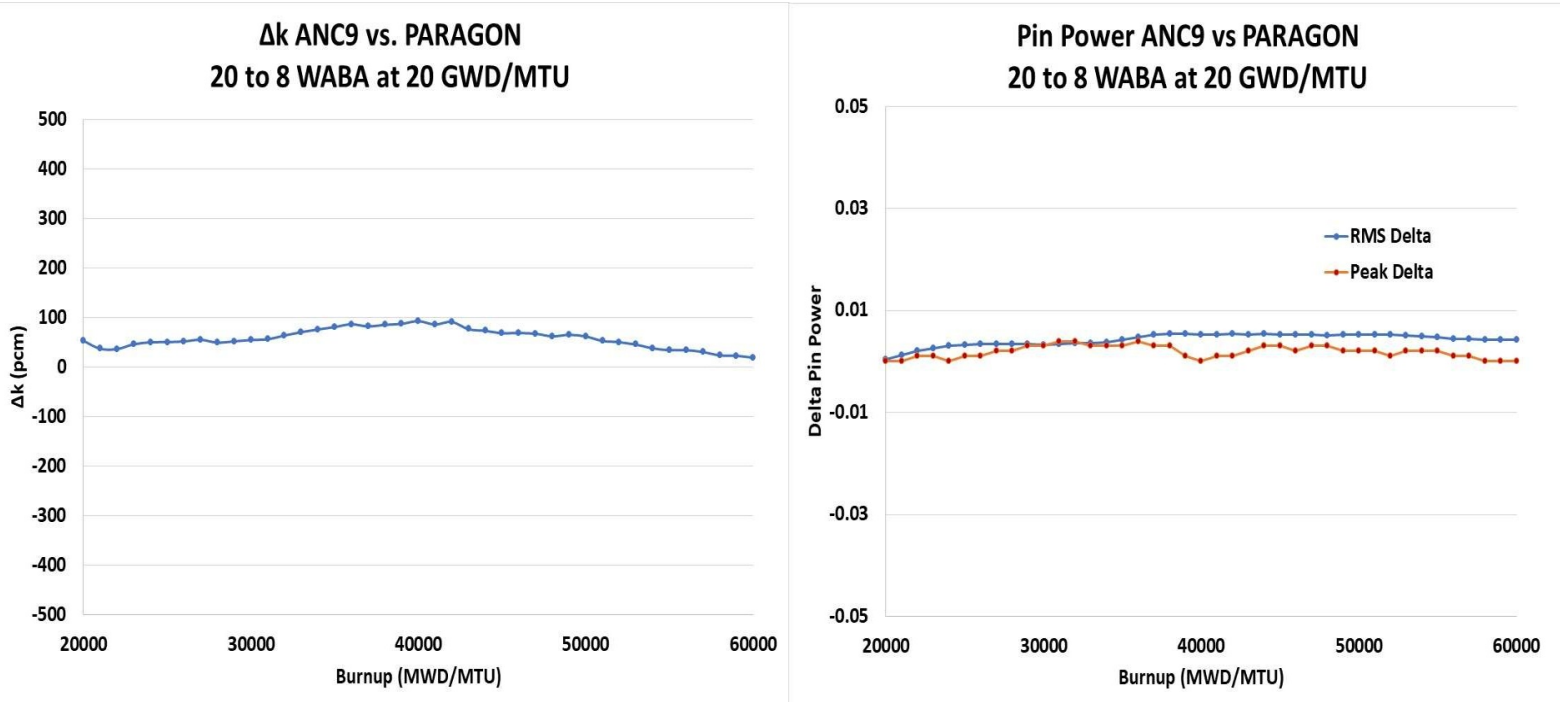

Figure 7. Comparison of eigenvalue and pin power for WABA replacement from 20 to 8 rods at 20 GWD/MTU
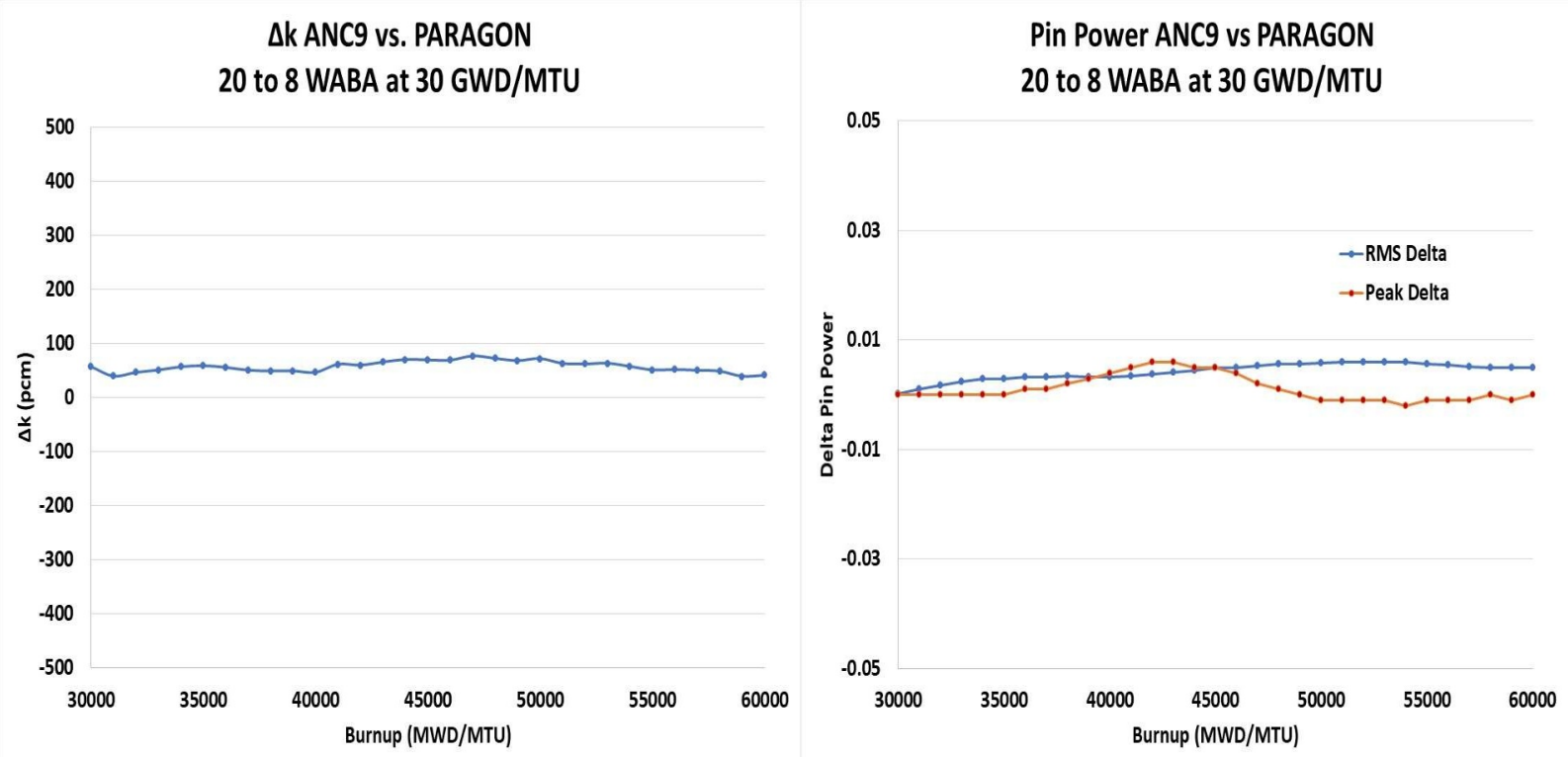

Figure 8. Comparison of eigenvalue and pin power for WABA replacement from 20 to 8 rods at 30 GWD/MTU

\section{CONCLUSIONS}

Generic Insert Methodology (GIM) has been developed and implemented in ANC9. This methodology is being qualified by modeling different absorber inserts into different fuel assemblies at different fuel burnup. By using "direct" and "indirect" feedback of absorber insert to macroscopic cross-sections, the insert 
depletion effect can be precisely captured by explicitly tracking the absorber isotopes of the insert and adoption of insert depletion correction.

ANC9 employed P3C methodology in pin power calculation, which follows individual fuel rod history and constructs both pin cell cross-sections and flux form factors based on the fuel burnup and spectrum history. This methodology naturally captures the absorber insert impact with any insertion scenarios. The qualification results show that GIM works as expected in the prediction of fuel reactivity and pin power distribution. Therefore, ANC9 with GIM is capable to perform the core design calculations and safety analysis for reload cores that involve different BP loading strategies.

\section{ACKNOWLEDGMENTS}

The authors would like to express our appreciation to Westinghouse Software Development and Test teams who provided support in this development. Thanks to Westinghouse technology user's group for their feedback.

\section{REFERENCES}

1. B, Zhang, et al, "Development of Generic Inserts Methodology", Proceedings of PHSOR 2016, Sun Valley, Idaho USA, May 1-5, 2016.

2. Y.S Liu, et al, ANC: "A Westinghouse Advanced Nodal Computer Code", WCAP-10966-A, WCAP-10966-A-AD1, Westinghouse Electric Company, September 1986, and 1989.

3. L. Mayhue, et al, "Qualification of NEXUS/ANC Nuclear Design System for PWR Analysis", Proceedings of PHSOR 2008, Interlaken, Switzerland, Sept. 14-19, 2008.

4. Y.S. Liu et al, "Pin Power Prediction in the Westinghouse Advanced Nodal Code", Trans. Am. Nuclear Soc., 53, 246, 1986.

5. B. Zhang, et al., "Development of a Three-Dimensional Pseudo Pin-by-Pin Calculation Methodology in ANC", Nuclear Technology, Volume 183 (3), September 2013.

6. M. Ouisloumen, et al. "PARAGON: The New Westinghouse Assembly Lattice Code", ANS International Meeting on Mathematical Methods for Nuclear Applications, Salt Lake City, Utah, USA, 2001. 